\title{
Development of forearm impedance plethysmography for the minimally invasive monitoring of cardiac pumping function
}

\author{
Jia-Jung Wang ${ }^{1}$, Wei-Chih $\mathrm{Hu}^{2}$, Tsiar Kao ${ }^{3}$, Chun-Peng Liu ${ }^{4}$, Shih-Kai Lin ${ }^{4}$ \\ ${ }^{1}$ Department of Biomedical Engineering, I-Shou University, Kaohsiung, Taiwan; \\ ${ }^{2}$ Department of Biomedical Engineering, Chung- Yuan Christian University, Chungli, Taiwan; \\ ${ }^{3}$ Department of Biomedical Engineering, Hungkuang University, Taichung, Taiwan; \\ ${ }^{4}$ Department of Internal Medicine, Kaohsiung Veterans General Hospital, Kaohsiung, Taiwan \\ Email: wangjj@isu.edu.tw; weichih@be.cycu.edu.tw; tskao@sunrise.hk.edu.tw; cpliu@vghks.gov.tw
}

Received 25 November 2010; revised 30 November 2010; accepted 3 December 2010.

\begin{abstract}
It is essential to continuously and non-invasively monitor the cardiac pumping function in clinical setting. Thus, the study aimed to explore a regional impedance phethysmographic method to assess the changes in stroke volume. To do this, we developed a plethysmographic device that was capable of delivering a single-frequency current with constant amplitude and of recording electrical impedance signals of biological tissue. The electrical impedance plethysmographic waveform form the lower arm was measured with the impedance plethysmographic device, and simultaneously the end-systolic and end- diastolic volumes of the left ventricle were obtained with a two-dimension echocardiographic system in fourteen healthy subjects before and immediately after a thirty-second breath-hold maneuver. For the 14 subjects, a linear correlation coefficient of $0.79(p<0.001)$ was obtained between the changes in peak amplitude of the forearm impedance waveform and the changes in stroke volume before and just after the breath-hold test. In addition, the changes in the mean area under the impedance curve and the change in stroke volume were also correlated linearly $(r=0.71, p<0.005)$. In summary, the forearm impedance plethysmography may be employed to evaluate the beat-to-beat alteration in cardiac stroke volume, suggesting its potential for long-term monitoring cardiac pumping performance.
\end{abstract}

Keywords: Impedance Plethysmography; Stroke Volume; Breath-Hold Maneuver; Impedance; Echocardiography

\section{INTRODUCTION}

The electrical impedance cardiography, one of the plethysmography, has been extensively applied to assess cardiac parameters, because of its minimal invasiveness of nature. The stroke volume, cardiac output, diastolic filling, ventricular contractility, and so forth, have been evaluated using this impedance plethysmography in previous literature [1-5]. According to the electrode arrangement and location, there are three most popular electrical impedance technologies applied for non-invasively assessing cardiac pumping function.

The first and the most commercially available is the thoracic electrical impedance cardiography, which utilizes a number of electrodes placed at the root of the neck and set around the lower part of the chest cage in the cardiac parameter measurement [6-8]. Since the electrodes arranged are close to the heart, the thoracic impedance cardiography may provide with more reliable cardiac parameter. However, the electrode location and arrangement makes it inconvenient and unwieldy in actual clinics. Second, the whole-body electrical impedance cardiography requires either four pairs of electrodes that are applied to four limbs, or two pairs of electrodes in which one pair is placed on a wrist and the other on the contralateral ankle [9-11]. Evidently, it is necessary for users to spend more time in appropriately disposing the eight electrodes in the whole-body impedance cardiography. Since the four limbs are concurrently measured with the technique, an impedance signal accompanied by more artifacts from the four extremity's motion will be yielded.

Third, the regional electrical impedance cardiography usually uses two current electrodes and two sensing electrodes that are placed on a local or regional position of a limb [3,12]. Previous reports have indicated that the regional impedance cardiography is as accurate as the thoracic or the whole-body electrical impedance cardiography $[2,13]$, with advantage of using peripheral rather than thoracic impedance waves.

The objective of the study is to propose a forearm 
impedance plethysmographic approach and to validate it by making a comparison between the changes and the per cent changes in peak amplitude of or mean area underneath the impedance wave from a forearm recorded with the plethysmography, and those in cardiac stroke volume measured with the echocardiography.

\section{METHODS}

\subsection{Theory of Impedance Plethysmography}

In the impedance plethysmography, the blood volume changes $\left(\Delta \mathrm{V}_{\mathrm{b}}\right)$ in relation to the changes in electrical impedance $(\Delta \mathrm{Z})$ can be governed by Nyboer's formula as follows [14]:

$$
\Delta V_{b}=\rho \frac{L^{2}}{Z_{0}^{2}} \Delta Z,
$$

where $\rho$ is the resistivity of blood, $L$ is the length between the voltage sensing electrodes and $Z_{0}$ is the initial value of the electrical impedance of the body segment. This formula can be cautiously modified to determine ventricular stroke volume from dZ/dt waveform [15]. Small variation in the instantaneous impedance of the forearm segment may rise from arterial and venous blood circulation, respiration, body motion, and so on. In the study, properly applied at desired location on the forearm surface, the measurement electrodes may response to the changes in the forearm impedance that is caused predominantly by the radial arterial blood flow.

\subsection{Impedance Measuring Apparatus}

An impedance measuring device with tetra-polar electrodes (ECG electrodes, Unomedical Ltd., Great Britain) was developed in the work to record the electrical impedance waveform in a lower arm. Figure 1 shows its schematic block diagram and the location of the electrodes on the medial surface of a forearm.

The impedance measuring device was composed of a Wein-Bridge oscillator designed to produce a sinusoidal wave with a frequency of $100 \mathrm{kHz}$. A voltage to current converter output a sinusoidal current of constant amplitude (less than $1 \mathrm{~mA}$ ) that acted as a carrier. The sinusoidal current could be passed through the forearm segment with the help of two spot electrodes called the current el (A and D). One of the two current electrodes was placed on a position as close to the wrist as possible, whereas the other was placed on a position close to the cubital fossa. Voltage signal generated along the current pathway was detected by means of another pair of spot electrodes called the voltage sensing electrodes or measurement electrodes ( $\mathrm{B}$ and $\mathrm{C}$ ). Between the two current electrodes, the two voltage sensing electrodes were placed over the radial artery.

An instrumentation amplifier (AD620AN) was applied

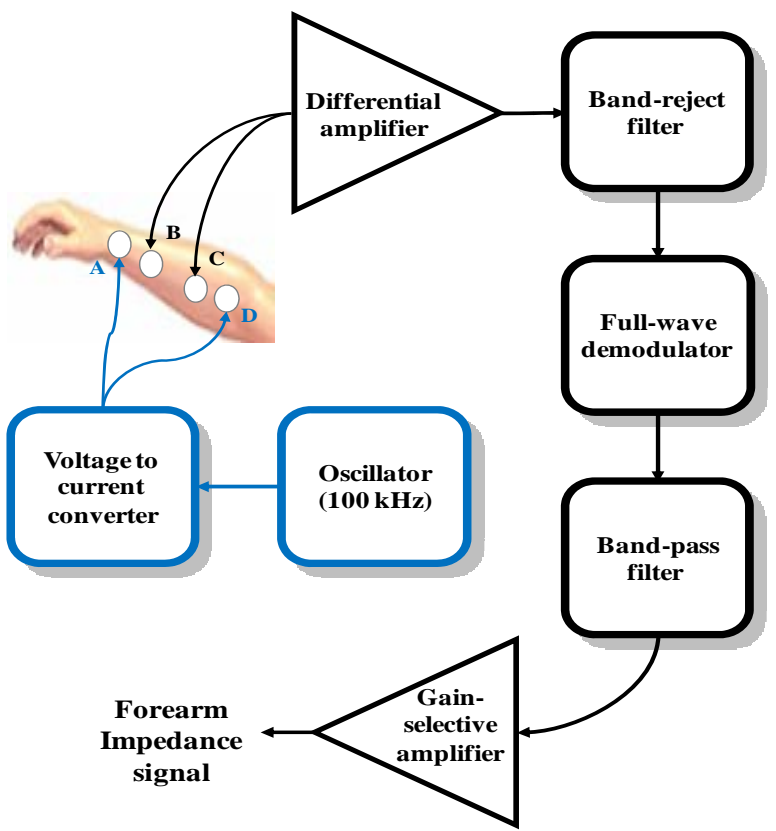

Figure 1. Block diagram of the impedance measuring device. Constant amplitude sinusoidal current is passed through the forearm segment by means of the current electrodes A and D. Along the current path, two voltage sensing electrodes B and C are appropriately applied to pick up the voltage that is proportional to instantaneous impedance of the forearm segment.

to pick up the voltage difference between the two measurement electrodes, due to its high input impedance. The gain of this instrumentation amplifier could be properly adjusted to output an alternating voltage signal whose amplitude was within $\pm 1 \mathrm{~V}$ and modulated by the $100 \mathrm{kHz}$ carrier. To increase the signal to noise ratio, a twin $\mathrm{T}$ band-rejection filter was used to significantly remove the superimposed noise (60 Hz noise) mostly arising from the power line. Moreover, we utilized a full-wave rectifying demodulator to rectify the alternating signal passing through the band-rejection filter. In addition, a four-order low-pass filter $(30 \mathrm{~Hz})$ and a one-order high-pass filter $(0.5 \mathrm{~Hz})$ of the band-pass circuit were used to demodulate the $100 \mathrm{kHz}$ carrier and to remove the dc bias and low-frequency drift, respectively. After the filtered signal was appropriately amplified by the gain-selective amplifier $(20,000$ to 200,000$)$, we could obtain the continuous impedance signal of the forearm segment.

\subsection{Breath-Hold Maneuver}

The experimental population included 14 male young subjects with an average age of $22.4 \pm 0.8$ years, an average height of $175.1 \pm 3.6 \mathrm{~cm}$, an average weight of $73.9 \pm 9.7 \mathrm{Kg}$, an average systolic blood pressure of $131.8 \pm 10.8$ (from 112 to 151 ) $\mathrm{mmHg}$, an average diastolic blood pressure of $75.1 \pm 7.6$ (from 66 to 89 ) 
$\mathrm{mmHg}$, and an average heart rate of $79.5 \pm 12.0$ (from 60 to 95 ) beats per minute. All the subjects had no cardiac disease and vascular stenosis. In order to non- invasively induce a considerable change in ventricular stroke volume, all the subjects were asked to undertake twice a thirty-second breath-hold maneuver in supine position. The Institutional Ethics Committee of the Kaohsiung Veterans General Hospital approved the study protocol and all subjects gave their consent.

\subsection{Stroke Volume Measurement with Echocardiography}

Based on the electrocardiogram signals, the left ventricular end-systolic and end-diastolic volumes of the 14 subjects in supine position were measured by means of a 2-dimension ultrasound system (SONOS-7500, Philips) at the Cardiovascular Center of the Kaohsiung Veterans General Hospital, Taiwan. The volume measurements with the echocardiography were performed in the two conditions: hemodynamic variable-stable condition (baseline) as well as immediately after a 30-second breath-hold maneuver. The stroke volume was defined as the difference between the end-diastolic and end-systolic volumes.

\subsection{Data Analysis}

Before extracting any values from the electrical impedance signals, the waveform was deliberately adjusted to set its minimum value to zero; that is, all values of the digitized data in the impedance waveform became larger than or equal to zero. The study employed ensemble averaging technique to minimize motion artifacts, external pick up and internal noise of the developed device. For individual subjects, a record containing 5 to 7 continuous cycles of the impedance waveform was used to determine the peak amplitude and the mean area beneath the impedance curve. To observe the alteration of one variable (M), such as the peak amplitude, mean area and stroke volume, in the pre- and post- 30-second breathhold experiment, the percent change in that variable $(\% \mathrm{M})$, can be calculated using the following equation,

$$
\% M=\frac{M_{a f t}-M_{0}}{M_{0}} \times 100 \%,
$$

where $\mathrm{M}_{0}$ and $\mathrm{M}_{\mathrm{aft}}$ are the variables measured before and just after the 30-second breath-hold maneuver, respectively.

The quantitative data were expressed as mean \pm STD . If a p value was less than 0.05 in paired t-test analysis, then the means of two variables measured before and just after the breath-hold maneuver were considered significantly different. To compare the changes and the percent changes in the peak amplitude of and the mean area under the impedance waveform with those in the stoke volume, 2-tailed Pearson's correlation is utilized.

\section{RESULTS}

A typical impedance waveform of the forearm segment recorded from one of the fourteen subjects is shown in Figure 2. The peak-to-peak amplitude of the impedance waveform was decreased in the end of the thirty-second breath-hold maneuver, as compared with the baseline. Besides, the peak-to-peak amplitude became noticeably greater immediately after the 30-second breath-hold maneuver than in the baseline condition. Even with superimposed noise, the impedance waveform showed a periodic fluctuation in a beat-to-beat manner. In the photo-plethysmographic waveform, its peak-to-peak amplitude also decreased in the end of the maneuver as compared with the baseline. Interestingly, the pattern of the photo-plethysmographic waveform during the simple breath-hold interval was similar to that during the Valsalva's maneuver.

Table 1 summarizes the parameters that are measured in the 14 subjects before and right away after the 30second breath-hold maneuver with the forearm impedance plethysmography and the 2-dimension echocardiography, respectively. The short-term breath-hold maneuver significantly increased the peak amplitude of the forearm impedance from $1.89 \pm 0.53$ to $2.26 \pm 0.42$ volt

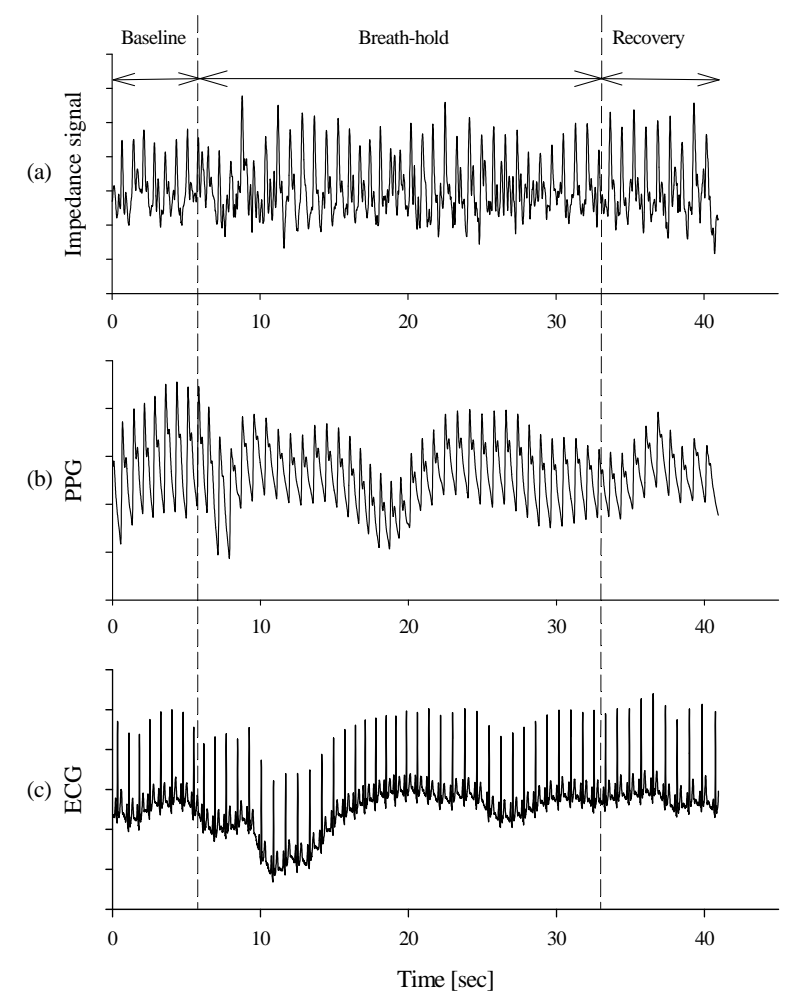

Figure 2. Time course of (a) electrical impedance signal measured from a forearm, (b) photo-plethysmographic (PPG) signal recorded from a fingertip of the same arm, and (c) electrocardiogram signal (ECG) from the lead-II. 
Table 1. Values of the parameters obtained with the forearm impedance plethysmogrphy as well as the echocardiography in the 14 subjects, respectively.

\begin{tabular}{|c|c|c|c|c|c|c|c|c|c|c|}
\hline \multirow{2}{*}{$\begin{array}{c}\text { Variable } \\
\text { Condition }\end{array}$} & \multicolumn{2}{|c|}{$\begin{array}{l}\text { Peak Amplitude } \\
\text { (volt) }\end{array}$} & \multicolumn{2}{|c|}{$\begin{array}{l}\text { Mean Area } \\
(\text { volt } \times \text { sec })\end{array}$} & \multicolumn{2}{|c|}{$\begin{array}{l}\text { ESV } \\
(\mathrm{ml})\end{array}$} & \multicolumn{2}{|c|}{$\begin{array}{l}\text { EDV } \\
(\mathrm{ml})\end{array}$} & \multicolumn{2}{|c|}{$\begin{array}{l}\text { SV } \\
(\mathrm{ml})\end{array}$} \\
\hline & $P R E$ & POST & $P R E$ & POST & $P R E$ & POST & PRE & POST & $P R E$ & POST \\
\hline Max & 2.86 & 3.11 & 1.112 & 1.106 & 54.9 & 52.5 & 124.0 & 124.0 & 69.4 & 71.5 \\
\hline Min & 1.25 & 1.61 & 0.281 & 0.296 & 25.0 & 21.7 & 62.9 & 76.1 & 37.9 & 46.1 \\
\hline Mean & 1.89 & 2.26 & 0.506 & 0.576 & 37.9 & 34.4 & 89.3 & 91.2 & 51.5 & 56.8 \\
\hline STD & 0.53 & 0.42 & 0.229 & 0.203 & 9.6 & 9.6 & 16.1 & 14.7 & 7.9 & 8.1 \\
\hline$P$ value & \multicolumn{2}{|c|}{$<0.002$} & \multicolumn{2}{|c|}{$<0.02$} & \multicolumn{2}{|c|}{$<0.002$} & \multicolumn{2}{|c|}{$<0.3$} & \multicolumn{2}{|c|}{$<0.004$} \\
\hline
\end{tabular}

$(\mathrm{p}<0.002)$. The mean area $(0.506 \pm 0.229$ volt $\times$ sec $)$ under the impedance waveform yielded before the maneuver was smaller than that $(0.576 \pm 0.203$ volt $\times$ sec $)$ immediately after the maneuver $(\mathrm{p}<0.02)$. In the echocardiographic measurement, the short-time breath-hold significantly decreased the ventricular end-systolic volume from $37.9 \pm 9.6$ to $34.4 \pm 9.6 \mathrm{ml}(\mathrm{p}<0.002)$. Interestingly, the end-diastolic volume had a tendency to decline just after the breath-hold maneuver, but with no significant difference as compared with the baseline. Furthermore, the cardiac stroke volume $(56.8 \pm 8.1 \mathrm{ml})$ instantly after the 30-second breath-hold maneuver became significantly greater than that $(51.5 \pm 7.9 \mathrm{ml})$ in the baseline condition $(\mathrm{p}<0.004)$.

The pooled data yielded before and immediately after the 30-second breath-hold maneuver were used to examine the linear relation between the ventricular stroke volume and the peak amplitude of electrical impedance of the forearm segment. As shown in Figure 3, the stroke volume tends to be positively proportional to the maximum amplitude of impedance, but correlates weakly with



Figure 3. Relationship between the peak amplitude of forearm impedance waveform and the stroke volume. The 28 data points in the plot are pooled ones of the 14 subjects measured before and immediately after the 30 -second breath-hold manipulation. the maximum amplitude in the subjects.

Changes in the maximum amplitude of forearm impedance with respect to changes in the cardiac stroke volume measured before and just after the 30-second breath-hold trial are plotted in Figure 4. Fascinatingly, the 14 subjects showed high correlation $(r=0.785$, $<0.001$ ) between the peak amplitude changes and the stroke volume changes. Likewise, between the baseline condition and the post-breath-hold maneuver, the percent change in the peak amplitude of forearm impedance linearly correlates well with the percent change in the stoke volume for the 14 subjects $(r=0.790, p<0.001)$, as shown in Figure 5.

The data measured in the control condition along with those measured instantly after the 30-second breath-hold trial were used together to investigate the linear relation between the mean area under the forearm impedance waveform and the ventricular stroke volume. Figure 6 shows that the mean area underneath the impedance waveform is hardly proportional to the cardiac stroke volume.

Figure 7 demonstrates the relation between the changes

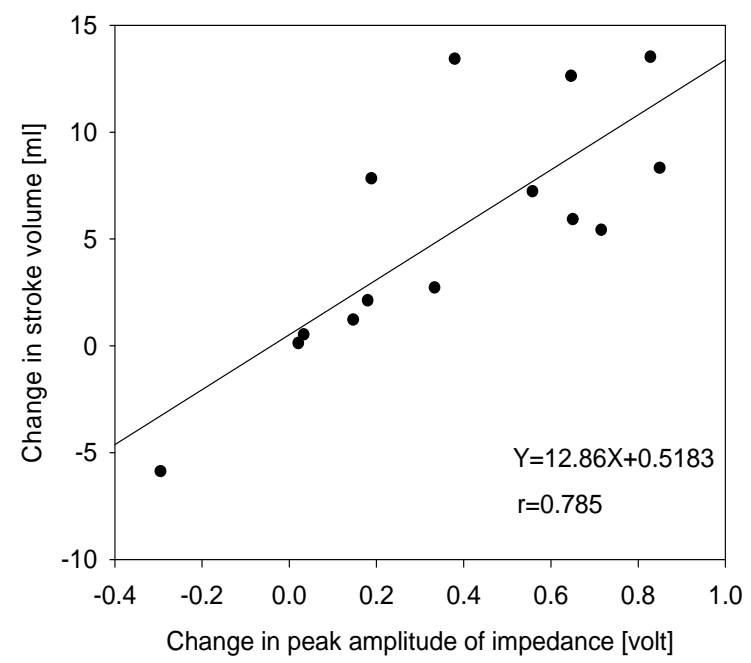

Figure 4. Relationship between the change in maximum amplitude of regional impedance waveform and the change in stroke volume before and just after the 30 -second breath-hold manipulation. 


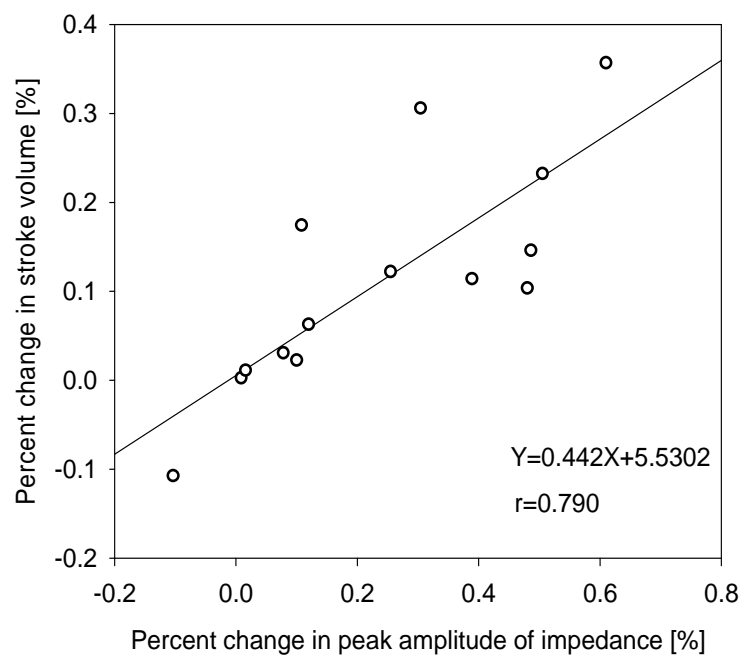

Figure 5. Relationship between the percent change in maximum amplitude of regional impedance waveform and the percent change in stroke volume before and just after the 30-second breath-hold manipulation.

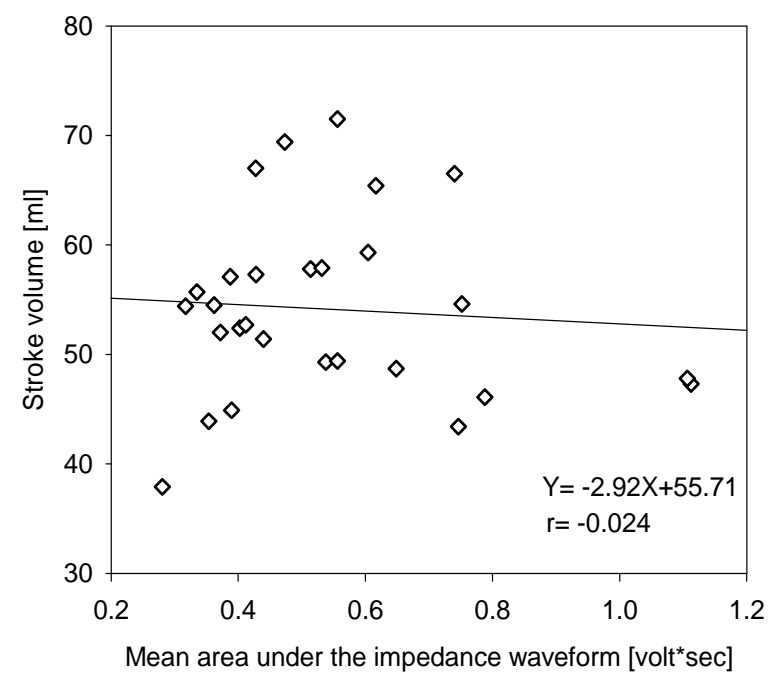

Figure 6. Relationship between the mean area under the forearm impedance waveform and the stroke volume in the 14 subjects using the data obtained before and just after the 30-second breath-hold manipulation.

in mean area under the impedance waveform and the changes in cardiac stroke volume measured before and just after the 30-second breath-hold experiment. It was found that there was a correlation coefficient of 0.715 $(p<0.005)$ between the peak amplitude changes and the stroke volume changes for the 14 subjects. As well, Figure 8 shows that between the baseline condition and the post-breath-hold maneuver, the percent change in the mean area under the forearm impedance waveform linearly correlates with the percent change in the stoke volume for the 14 subjects $(r=0.704, p<0.005)$.

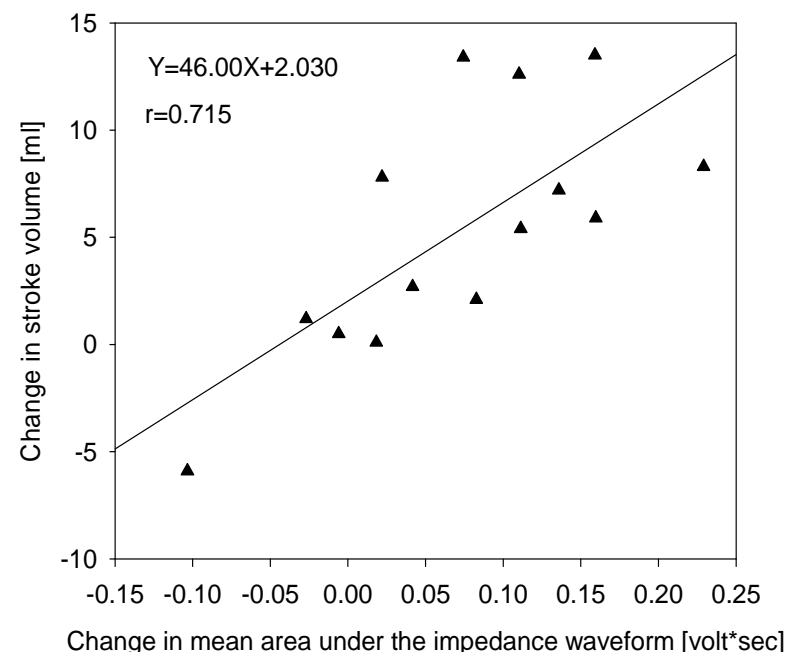

Figure 7. Relationship between the change in mean area under the forearm impedance waveform and the change in stroke volume before and just after the 30-second breath-hold manipulation.

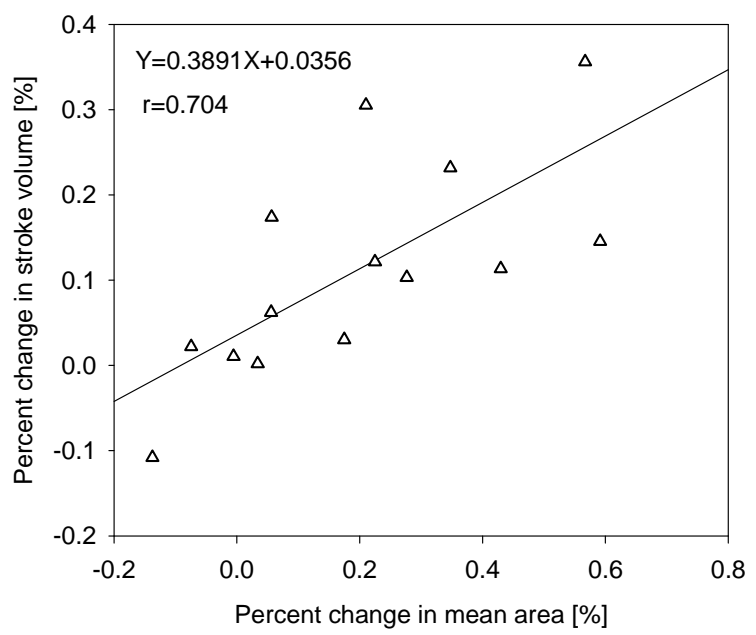

Figure 8. Relationship between the percent change in mean area under the forearm impedance waveform and the percent change in stroke volume before and just after the 30-second breath-hold manipulation.

\section{DISCUSSION}

Several previous studies have reported the potential usefulness of the whole-body or the thoracic impedance cardiography in the evaluation of stroke volume in patients with different diseases or clinical situations $[2,16,17]$. However, these two kinds of cardiography require more pairs of electrodes and spend more time in placing the electrodes on desired locations appropriately. On the other hand, in the proposed forearm impedance plethysmography, less pairs of the electrodes are needed and only the medial surface of a forearm is used as the 
measurement region. This may make the proposed method more suitable for actually clinical applications.

In the whole-body and thoracic impedance cardiography, an electrical current delivered by a current source should pass through the core body or at least the thoracic tissue. Thus, it is hardly to describe the actual current pathway. Also, a portion of the delivered current may really go through the heart, perhaps resulting in a risk of microshock. In contrast, the current sent out by the developed device in the study only passes through the forearm segment, without possibility of cardiac microshock.

In accordance with the Kirchov's law, electrical current tends to pass through human tissue with higher conductivity. If an alternating current with a frequency of $100 \mathrm{KHz}$ is here applied to the forearm segment, both the radial arterial lumen filled with blood and the extracellular fluid should gain higher current density, due to their lower resistance [18]. Therefore, when the two measurement electrodes are deliberately placed just over the radial artery, the voltage between the sensing electrodes is proportional to the forearm segment impedance. Since the forearm impedance follows the radial arterial blood flow. So, the change in the voltage chiefly reflects that in the radial arterial blood flow. To demonstrate this, we first put a pneumatic cuff around the upper arm and then inflate it up to different pressure levels. When the internal cuff pressure is increased up to about $60 \mathrm{mmHg}$, the blood flow in the veins inside the upper arm will possibly stop, leading to little change in the amplitude of the electrical impedance waveform in the lower arm. But, when the cuff is inflated to the mean arterial pressure resulting in a partially occlusion in the brachial artery of underlying, a moderate reduction in the impedance amplitude is found. As the cuff is further increased up to a supra-systolic pressure level, the impedance amplitude is significantly decreased, as shown in Figure 9.

It is assumed in the study that the amount of blood flow through the radial artery of interest is linearly proportional to the stroke volume produced by the left ventricle in stable conditions. Consequently, the change in the radial arterial flow may correlate linearly with the change in the stroke volume. Based on the above description, the change in the amplitude of as well as the change in the area under the forearm impedance waveform might be proportional to that in the stroke volume. The present data in Figures 4 and 7 are likely to support this hypothesis. That might be partially the reason that the regional impedance cardiography may provide more accuracy than the thoracic or the whole-body cardiography in assessing the stroke volume [3].

To accurately determine the subject's stroke volume of the patients with different diseases or clinical situations using the whole-body impedance cardiography all the times becomes controversial [19,20], because the electrical current in this approach passes through more

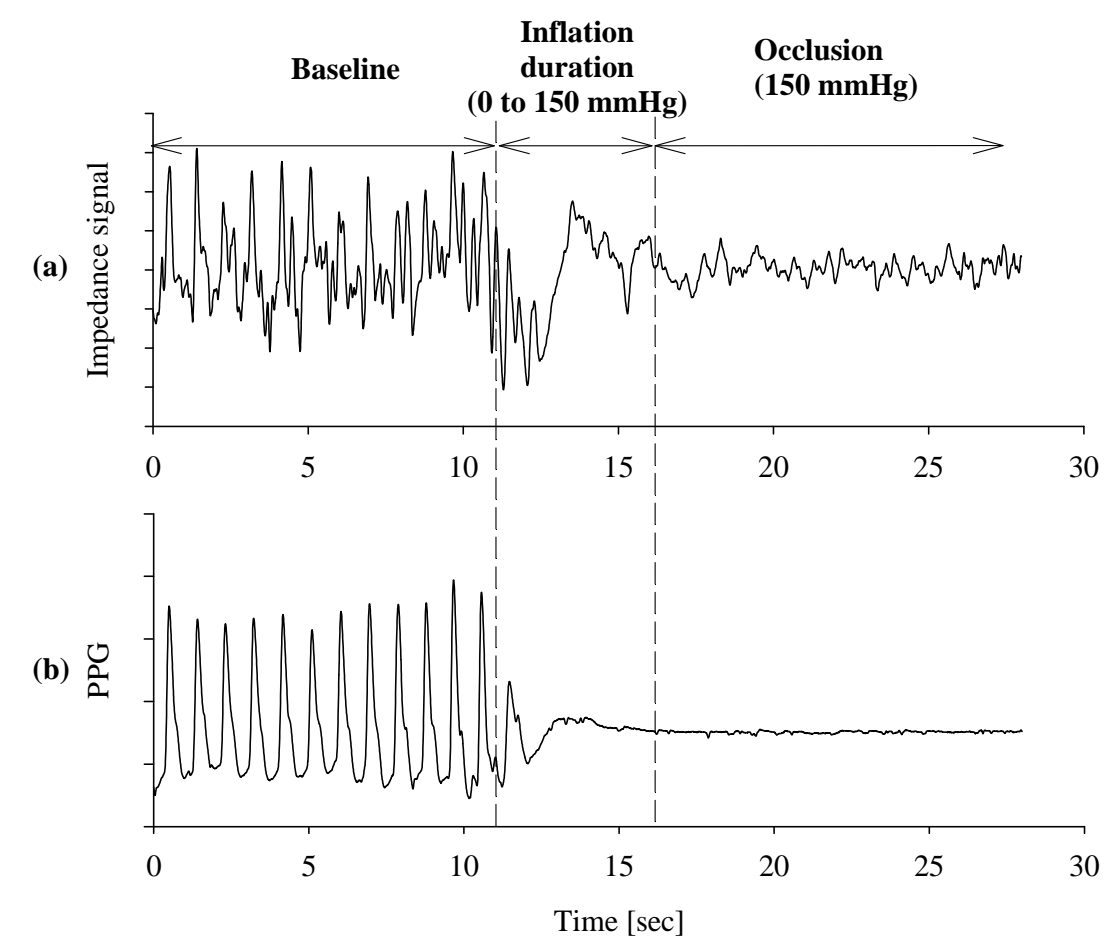

Figure 9. (a) Fluctuation of impedance amplitude becomes smaller when the upper arm is pressurized to be $150 \mathrm{mmHg}$. (b) Photo-plethysmographic signal simultaneously recorded from a fingertip in the same arm. 
complicated conductive components, compared to the present study. In the present study, we apply an alternating current of $100 \mathrm{KHz}$ to the circle-type current electrodes placed directly on the top of the radial artery, which may result in a higher current density in the radial lumen. Thus, the change in the forearm impedance amplitude may probably follow the change in the radial blood flow. It suggests that the change in the impedance amplitude can be more specifically reflected the change in the stroke volume.

Several factors associated with the impedance changes are present between individuals. One is the position of surface electrodes. In most cases, the distance between the two inner or outer electrodes is different from each other in the measurement situation, resulting in a considerable change in impedance amplitude. Second is due to the distinct diameters of radial arteries and different peripheral tissue compositions. Third is related to the variation in biological impedance of tissue of interest. Unfortunately, the present device fails to directly determine the absolute stroke volume. Our results show a correlation coefficient of less than 0.3 between the maximum impedance amplitude and stroke volume, with a similar low coefficient between the underneath area of the impedance wave and the stroke volume.

\section{CONCLUSION}

Linear relationships between the changes in amplitude of and in the area under the forearm impedance waveform and the changes in stroke volume are shown. Thus, the forearm impedance plethysmography proposed may be utilized to assess the beat-to-beat change in cardiac stroke volume, suggesting its potential for long-term monitoring ventricular pumping function.

\section{ACKNOWLEDGEMENTS}

This study was supported by the National Science Council, Taiwan, the Republic of China, under grant numbers NSC 98-2221-E-214-005MY3 and NSC 98-2221-E-075B-001-MY2.

\section{REFERENCES}

[1] Absi, M.A., Lutterman, J. and Wetzel, G.T. (2010) Noninvasive cardiac output monitoring in the pediatric cardiac Intensive Care Unit. Current Opinion in Cardiology, 25, 77-79. doi:10.1097/HCO.0b013e3283362452

[2] Cohen, A.J., Arnaudov, D., Zabeeda, D., Schultheis, L., Lashinger, J. and Schachner, A. (1998) Non-invasive measurement of cardiac output during coronary artery bypass grafting. European Journal of Cardio-Thoracic Surgery, 14, 64-69. doi:10.1016/S1010-7940(98)00135-3

[3] Cotter, G., Schachner, A., Sasson, L., Dekel, H. and Moshkovitz, Y. (2006) Impedance cardiography revisited.
Physiological Measurement, 27, 817-827. doi:10.1088/0967-3334/27/9/005

[4] Peng, Z.Y., Critchley, L.A., Fok, B.S. and James, A.E. (2004) Evaluation of impedance based indices of cardiac contractility in dogs. Journal of Clinical Monitoring and Computing, 18, 103-109. doi:10.1023/B:JOCM.0000032720.02801.e6

[5] Woltjer, H.H., Bogaard, H.J., Scheffer, G.J., van der Spoel, H.I., Huybregts, M.A. and de Vries, P.M. (1996) Standardization of non-invasive impedance cardiography for assessment of stroke volume: comparison with thermodilution. British Journal of Anaesthesia, 77, 748-752.

[6] Atallah, M.M. and Demain, A.D. (1995) Cardiac output measurement: lack of agreement between thermodilution and thoracic electric bioimpedance in two clinical settings. Journal of Clinical Anesthesia, 7, 182-185. doi:10.1016/0952-8180(94)00050-E

[7] Hahn, G., Sipinkova, I., Baisch, F. and Hellige, G. (1995) Changes in the thoracic impedance distribution under different ventilatory conditions. Physiological Measurement, 16, A161-A173.

doi:10.1088/0967-3334/16/3A/016

[8] Imhoff, M., Lehner, J.H. and Lohlein, D. (2000) Noninvasive whole-body electrical bioimpedance cardiac output and invasive thermodilution cardiac output in high-risk surgical patients. Critical Care Medicine, 28, 2812-2818. doi:10.1097/00003246-200008000-00022

[9] Kubicek, W.G., Kottke, J., Ramos, M.U., Patterson, R.P., Witsoe, D.A., Labree, J.W., Remole, W., Layman, T.E., Schoening, H. and Garamela, J. T. (1974) The Minnesota impedance cardiograph-theory and applications. Biomedical Engineering, 9, 410-416.

[10] Leitman, M., Sucher, E., Kaluski, E., Wolf, R., Peleg, E., Moshkovitz, Y., Milo-Cotter, O., Vered, Z. and Cotter, G. (2006) Non-invasive measurement of cardiac output by whole-body bio-impedance during dobutamine stress echocardiography: clinical implications in patients with left ventricular dysfunction and ischaemia. European Journal of Heart Failure, 8, 136-140. doi:10.1016/j.ejheart.2005.06.006

[11] Cotter, G., Moshkovitz, Y., Kaluski, E., Cohen, A., Miller, J. H., Goor, D. and Vered, Z. (2004) Accurate, noninvasive continuous monitoring of cardiac output by whole-body electrical bioimpedance. Chest, 125, 1431-1440. doi:10.1378/chest.125.4.1431

[12] Critchley, L.A., Peng, Z.Y., Fok, B.S. and James, A.E. (2005) The effect of peripheral resistance on impedance cardiography measurements in the anesthetized dog. Anesthesia and Analgesia, 100, 1708-1712. doi:10.1213/01.ANE.0000150602.40554.EB

[13] Wong, K.L. and Hou, P.C. (1996) The accuracy of bioimpedance cardiography in the measurement of cardiac output in comparison with thermodilution method. Acta Anaesthesiologica Sinica, 34, 55-59.

[14] Nyboer, J. (1960) Regional pulse volume and perfusion flow measurements: electrical impedance plethysmography. Archives of Internal Medicine, 105, 264-276.

[15] Patterson, R.P., Wang, L. and Raza, S.B. (1991) Impedance cardiography using band and regional electrodes in supine, sitting, and during exercise. IEEE 
Transactions on Biomedical Engineering, 38, 393-400. doi:10.1109/10.81557

[16] Adamicza, A., Tutsek, L., Daroczy, B., Bari, F. and Nagy, S. (1994) The measurement of cardiac output in dogs by impedance cardiography with different electrode arrangements. Acta Physiological Hungarica, 82, 37-52.

[17] Paredes, O.L., Shite, J., Shinke, T., Watanabe, S., Otake, H., Matsumoto, D., Imuro, Y., Ogasawara, D., Sawada, T. and Yokoyama, M. (2006) Impedance cardiography for cardiac output estimation: reliability of wrist-to-ankle electrode configuration. Circulation Journal, 70, 1164-1168. doi:10.1253/circj.70.1164

[18] Wtorek, J. and Plinski, A. (2005) The contribution of blood-flow-induced conductivity changes to measured impedance," IEEE Transactions on Biomedical Enginee- ring, 52, 41-49. doi:10.1109/TBME.2004.839633

[19] Miles, D.S., Gotshall, R.W., Quinones, J.D., Wulfeck, D.W. and Kreitzer, R.D. (1990) Impedance cardiography fails to measure accurately left ventricular ejection fraction. Critical Care Medicine, 18, 221-228. doi:10.1097/00003246-199002000-00019

[20] Stout, C.L., Van de Water, J.M., Thompson, W.M., Bowers, E.W., Sheppard, S.W., Tewari, A.M. and Dalton M.L. (2006) Impedance cardiography: can it replace thermodilution and the pulmonary artery catheter? The American Surgeon, 72, 728-732. 